\title{
Dinâmica de decomposição e liberação de nutrientes de genótipos de guandu sob cultivo em aléias
}

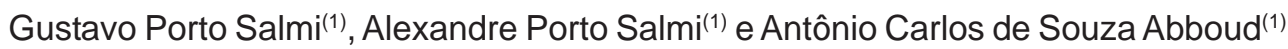

\begin{abstract}
(1)Universidade Federal Rural do Rio de Janeiro, Instituto de Agronomia, Dep. de Fitotecnia, BR 465, Km 7, CEP 23890-000 Seropédica, RJ. E-mail: gustavosalmi@ig.com.br, asalmi@bol.com.br, abboud@ufrrj.br
\end{abstract}

\begin{abstract}
Resumo - O objetivo deste trabalho foi avaliar a produção de fitomassa aérea, seus teores de N, P, K, e a dinâmica de liberação desses nutrientes, em seis genótipos de guandu (Cajanus cajan), em sistema de cultivo em aléias. A produção média de biomassa foi de 5,9 $\mathrm{Mg} \mathrm{ha}^{-1}$; o acúmulo de $\mathrm{N}$ variou de 188,3 a 261,3 $\mathrm{kg} \mathrm{ha}^{-1}$, o de $\mathrm{P}$ de 7,2 a 9,4 kg ha-1 e o de $\mathrm{K}$ de 29,3 a 45,5 kg ha-1; não houve diferença estatística entre os genótipos avaliados. As curvas de liberação mostraram que, aos 56 dias aproximadamente 60\% do N e 65\% do P e do K, contidos na biomassa remanescente, haviam sido liberados ao solo. Não houve efeito dos genótipos sobre a taxa de liberação de nutrientes. Os resultados indicam que os seis genótipos estudados foram igualmente eficientes em produzir biomassa e em liberar nutrientes de modo satisfatório, no primeiro ano de estudos.
\end{abstract}

Termos para indexação: Cajanus cajan, adubação verde, biomassa, nitrogênio, fósforo e potássio.

\section{Decomposition dynamics and nutrient release by pigeon pea genotypes under alley cropping}

\begin{abstract}
The objective of this research was to evaluate the aerial biomass, N, P and K contents, and the dynamics of decomposition and mineralization, in six genotypes of pigeon pea, grown as hedgerows in an alley cropping system. Dry biomass production averaged 5.9 $\mathrm{Mg} \mathrm{ha}^{-1}$; N content ranged from 188.3 to $261.3 \mathrm{~kg} \mathrm{ha}^{-1}$; P content, from 7.2 to $9.4 \mathrm{~kg} \mathrm{ha}^{-1}$ and $\mathrm{K}$ content, from 29.3 to $45.5 \mathrm{~kg} \mathrm{ha}^{-1}$. No differences between genotypes were found. Decay curves indicated a rate of $75 \%$ of the biomass still remaining 30 days after deposition. Also, approximately $60 \%$ of $\mathrm{N}$, and $65 \%$ of $\mathrm{P}$ and $\mathrm{K}$, of the remaining biomass, were released to soil. No effects of pigeon pea genotypes were found in decomposition or mineralization rates.
\end{abstract}

Index terms: Cajanus cajan, green manuring, biomass, nitrogen, phosphorus, potassium.

\section{Introdução}

Nas últimas décadas, novos conceitos de sistemas de produção agrícola, baseados na conservação do solo, diversificação de culturas, reciclagem de nutrientes, uso sistemático de adubos orgânicos e outras práticas alternativas, têm sido desenvolvidos na tentativa de equilibrar a produtividade com a conservação do meio ambiente. Uma das modalidades de produção que atende a esse conceito é o sistema de cultivo em aléias. Kang et al. (1981) definiram-no como um sistema de cultivo comercial, entre faixas de arbustos ou árvores, as quais são periodicamente podadas durante o ciclo da cultura, para controlar a sombra e prover material orgânico e nutrientes minerais para a cultura principal.
Há, no Brasil, carência de informações sobre esse sistema para produção das diversas culturas econômicas. O guandu (Cajanus cajan (L.) Millsp.) tem se mostrado uma excelente leguminosa para inclusão em sistema de cultivo em aléia, que pode produzir até $11 \mathrm{t} \mathrm{ha}^{-1}$ de fitomassa seca, o que pode incorporar ao sistema até $283 \mathrm{~kg} \mathrm{ha}^{-1}$ de N e $23 \mathrm{~kg} \mathrm{ha}^{-1}$ de P (Moreira, 2003; Alves et al., 2004). Com a crescente demanda de tecnologias que utilizam insumos biológicos com recursos locais, passou a ser ainda mais importante conhecer a produtividade de biomassa e os seus teores de N, P e K, bem como a dinâmica da liberação desses nutrientes ao solo, por diferentes genótipos de guandu, para se aproveitar a enorme diversidade biológica dessa espécie, sub-explorada no Brasil e se obter resultados mais eficientes desse sistema de produção. 


\section{Material e Métodos}

O experimento foi instalado em um Planossolo, na área do Campo Experimental do Dep. de Fitotecnia, da Universidade Federal Rural do Rio de Janeiro, no Município de Seropédica, RJ. A área experimental está situada a $22^{\circ} 45^{\prime} \mathrm{S}, 43^{\circ} 41^{\prime} \mathrm{W}$ e $35-40 \mathrm{~m}$ de altitude (Silva, 1993). A análise química de solo apresentou os seguintes valores: pH (água), 5,8; carbono orgânico, $3,9 \mathrm{~g} \mathrm{~kg}^{-1} ; \mathrm{Al}^{3+}, 0,0 \mathrm{cmol}_{\mathrm{C}} \mathrm{dm}^{-3} ; \mathrm{Ca}^{2+}, 1,6 \mathrm{cmol}_{\mathrm{C}} \mathrm{dm}^{-3}$; $\mathrm{Mg}^{2+}, 0,6 \mathrm{cmol}_{\mathrm{c}} \mathrm{dm}^{-3} ; \mathrm{P}, 13,6 \mathrm{mg} \mathrm{dm}^{-3}$; e $\mathrm{K}^{+}$, $16 \mathrm{mg} \mathrm{dm}^{-3}$. O experimento foi instalado em outubro de 2003 e constou do plantio de faixas simples de guandu, isto é, linhas individuais, com espaçamento de $0,5 \mathrm{~m}$ entre plantas. As faixas que constituíram as aléias foram espaçadas de $4 \mathrm{~m}$. Foram testados seis genótipos de guandu: Brejo Santo, G-35, Barbalha Arajará, ICP 8518, Cariri Açu e Triunfo P.J., todos provenientes do Banco de Germoplasma da Embrapa Semi-Árido. Os genótipos não sofreram adubação nem inoculação.

Por ocasião do florescimento, ocorrido em março de 2004, as plantas foram podadas à altura de $100 \mathrm{~cm}$ do solo, e a fitomassa foi depositada em cobertura nas aléias, ao lado de cada faixa.

As variáveis avaliadas foram a produção de fitomassa, depois da poda, a sua dinâmica de decomposição, os teores de N, P e K, incluindo as perdas desses nutrientes.

Para se estimar a produção de fitomassa fresca, três plantas do centro de cada parcela foram cortadas, na altura de $100 \mathrm{~cm}$, pesadas no campo, e levadas ao laboratório, onde se determinou a relação de folhas e de ramos. Um grupo de subamostras foi formado, mantendo-se a relação folhas:ramos originais, e foram acondicionadas em sacos de papel e levadas para secar em estufa de circulação forçada, a $65^{\circ} \mathrm{C}$, até massa constante, para determinação da massa da matéria seca.

No segundo grupo de subamostras, procedeu-se o estudo da dinâmica de decomposição da biomassa e liberação de nutrientes in situ, pelo método de sacola de serapilheira (Anderson \& Ingram, 1996), por um período de 56 dias, a partir da poda. A fitomassa foi seccionada em fragmentos de $5 \mathrm{~cm}$ e, de cada parcela, foram preparados 20 lotes, com 50 g de tecido vegetal cada. Uma subamostra foi, também, secada em estufa de ar forçado, a $65^{\circ} \mathrm{C}$, até massa constante, para se determinar a massa do tecido vegetal no início da decomposição (To). Cada um dos 20 lotes foi acondicionado em bolsas de náilon, com dimensão de $25 \times 25$ cm, con- feccionadas com telas de abertura de malha de $0,4 \mathrm{~mm}$, para acesso da fauna edáfica. As bolsas foram dispostas à superfície do solo, cada qual na parcela de onde foi cortada, e retiradas aos 7, 14, 28, 42 e 56 dias, depois do corte das plantas. Foram usadas quatro repetições por data de coleta. Em cada uma dessas épocas, o resíduo vegetal de cada bolsa foi limpo manualmente e secado em estufa, à temperatura de $65^{\circ} \mathrm{C}$, até alcançar massa constante, e, em seguida, moído. Foram avaliados a matéria seca e os teores de $\mathrm{N}, \mathrm{P}$ e $\mathrm{K}$, do tecido remanescente na sacola, em cada época.

O procedimento para análise de $\mathrm{N}$ baseou-se no método recomendado por Tedesco et al. (1995). Nos mesmos extratos mineralizados, foram determinados os teores de $\mathrm{P}$ e $\mathrm{K}$, pelo método colorimétrico vanadato-molibdato (Tedesco et al., 1995), e por espectrofotometria de chama (Embrapa, 1979), respectivamente. Os valores obtidos foram transformados em porcentagem relativa à massa e ao teor de nutrientes do início da decomposição (To). Com esses dados, foram determinadas as taxas de decomposição da biomassa e de liberação de nutrientes, para cada um dos tratamentos, utilizando-se o modelo matemático exponencial, descrito por Thomas \& Asakawa (1993): C = Coe-kt, em que $C$ é a quantidade de biomassa seca, ou nutrientes remanescentes, depois de um período de tempo t, em dias; Co refere-se à quantidade de matéria seca no início da decomposição; $\mathrm{k}$ é a constante de decomposição.

O tempo de meia vida $\left(T_{1 / 2}\right)$, ou seja, o tempo necessário para ser liberada a metade dos nutrientes da biomassa vegetal existente no tempo inicial, foi calculado a partir dos valores de $\mathrm{k}$ do modelo matemático, em que: $\mathrm{T}_{1 / 2}=\operatorname{Ln} 0,5 \mathrm{k}^{-1}$.

Na análise de variância, a comparação entre as médias de cada genótipo foi realizada pelo teste de Tukey, a $5 \%$ de probabilidade.

\section{Resultados e Discussão}

O rendimento de fitomassa aérea, na avaliação realizada 170 dias depois da semeadura, foi semelhante para os seis genótipos de guandu (Tabela 1), tendo variado de 14,15 a 18,31 $\mathrm{Mg} \mathrm{ha}^{-1}$ de matéria verde e de 4,67 a 5,95 $\mathrm{Mg} \mathrm{ha}^{-1}$ de matéria seca. Os valores de biomassa seca encontrados foram inferiores aos valores observados por Alves et al. (2004), que trabalharam com faixas duplas de guandu, na mesma região, e obtiveram rendimentos de $11 \mathrm{Mg} \mathrm{ha}^{-1}$. 
Conforme Wutke (1987), a altura de corte de $100 \mathrm{~cm}$ da superfície do solo resultou em rendimentos altos de biomassa, contribuindo para isso os pesos dos segmentos diferenciados de caule e de alguns ramos. Segundo Bahar \& Prine (1982), o rendimento de fitomassa de dez genótipos de guandu, podados à altura de 50 e $25 \mathrm{~cm}$, em duas estações diferentes, foram, em média, $5,4 \mathrm{Mg} \mathrm{ha}^{-1}$ para $50 \mathrm{~cm}$ e 6,25 $\mathrm{Mg} \mathrm{ha}^{-1}$ para $25 \mathrm{~cm}$ de altura. O mesmo experimento, com três podas de $25 \mathrm{~cm}$, produziu $46,6 \mathrm{Mg} \mathrm{ha}^{-1}$, e com quatro podas a $50 \mathrm{~cm}$, 45,3 $\mathrm{Mg} \mathrm{ha}^{-1}$.

Quando se objetiva o rendimento econômico de biomassa verde em cortes sucessivos, como é o caso do cultivo em aléias, deve-se considerar o aspecto de rebrota e de sobrevivência das plantas remanescentes, pois ambas as características são favorecidas por alturas de corte mais elevadas, (Otero, 1961; Viana \& Albuquerque, 1969; Akinola \& Whiteman, 1975; Wutke, 1987). Conforme a finalidade de utilização da biomassa produzida, alturas de corte menores devem ser preferidas, quando as plantas forem destinadas a um único corte, pois resultariam maiores rendimentos de biomassa (Wutke, 1987).

Os teores encontrados de N, P e K, na biomassa aérea do guandu (Tabela 2), indicam valores de N e P den-

Tabela 1. Produção de biomassa da parte aérea $\left(\mathrm{Mg} \mathrm{ha}^{-1}\right)$ de genótipos de guandu, nas faixas do experimento em aléia(1).

\begin{tabular}{lcc}
\hline Genótipo & Biomassa verde & Biomassa seca \\
\hline Barbalha Arajará & $15,89 \mathrm{a}$ & $4,98 \mathrm{a}$ \\
Brejo Santo & $18,31 \mathrm{a}$ & $5,95 \mathrm{a}$ \\
G-35 & $16,64 \mathrm{a}$ & $5,17 \mathrm{a}$ \\
Cariri Açu & $14,55 \mathrm{a}$ & $4,85 \mathrm{a}$ \\
ICP 8518 & $14,98 \mathrm{a}$ & $5,10 \mathrm{a}$ \\
Triunfo P.J. & $14,15 \mathrm{a}$ & $4,67 \mathrm{a}$ \\
\hline CV $(\%)$ & 36,63 & 38,35 \\
\hline
\end{tabular}

(1)Médias seguidas da mesma letra, nas colunas, não diferem entre si pelo teste de Tukey a 5\% de probabilidade.

Tabela 2. Concentração e acúmulo de nutrientes, na fitomassa de genótipos de guandu proveniente de poda, na época do florescimento ${ }^{(1)}$.

\begin{tabular}{|c|c|c|c|c|c|c|}
\hline \multirow[t]{2}{*}{ Genótipo } & \multicolumn{2}{|c|}{$\mathrm{N}$} & \multicolumn{2}{|r|}{$\underline{P}$} & \multicolumn{2}{|c|}{$\mathrm{K}$} \\
\hline & $(\%)$ & $\left(\mathrm{kg} \mathrm{ha}^{-1}\right)$ & $(\%)$ & $\left(\mathrm{kg} \mathrm{ha}^{-1}\right)$ & $(\%)$ & $\left(\mathrm{kg} \mathrm{ha}^{-1}\right)$ \\
\hline Barbalha Arajará & $3,78 b$ & $188,26 a$ & $0,14 a$ & $7,19 \mathrm{a}$ & $0,71 \mathrm{a}$ & $35,43 a$ \\
\hline Brejo Santo & $4,38 \mathrm{a}$ & $261,33 a$ & $0,15 \mathrm{a}$ & $9,41 \mathrm{a}$ & $0,76 \mathrm{a}$ & $45,48 \mathrm{a}$ \\
\hline G-35 & $4,05 \mathrm{ab}$ & $209,80 a$ & $0,16 a$ & $8,39 a$ & $0,58 \mathrm{a}$ & $30,32 a$ \\
\hline Cariri Açu & $3,91 \mathrm{~b}$ & $190,08 \mathrm{a}$ & $0,15 \mathrm{a}$ & $7,56 \mathrm{a}$ & $0,68 \mathrm{a}$ & $33,31 \mathrm{a}$ \\
\hline ICP 8518 & $4,02 \mathrm{ab}$ & $205,25 \mathrm{a}$ & $0,16 \mathrm{a}$ & $8,50 \mathrm{a}$ & $0,57 \mathrm{a}$ & $29,27 \mathrm{a}$ \\
\hline Triunfo P.J. & $4,15 \mathrm{ab}$ & $194,09 \mathrm{a}$ & $0,16 \mathrm{a}$ & $7,93 \mathrm{a}$ & $0,66 a$ & $31,10 \mathrm{a}$ \\
\hline $\mathrm{CV}(\%)$ & 3,84 & 35,88 & 6,43 & 8,75 & 13,21 & 34,79 \\
\hline
\end{tabular}

${ }^{(1)}$ Médias seguidas da mesma letra, nas colunas, não diferem entre si pelo teste de Tukey a 5\% de probabilidade. tro das faixas encontradas por outros autores, porém os teores de $\mathrm{K}$, foram mais baixos que aqueles encontrados por Souza \& Resende (2003), provavelmente decorrente dos baixos teores de $\mathrm{K}$ no solo.

Maiores porcentuais de $\mathrm{N}$ foram encontrados nos genótipos Brejo Santo, Triunfo P.J., G-35 e ICP 8518, todos acima de $4 \%$, enquanto, nos demais, os valores foram menores. As diferenças entre genótipos de guandu não foram significativas, apesar de as variações terem sido numericamente expressivas, como para acúmulo de $\mathrm{N}$, que variou de 188,26 a $261,33 \mathrm{~kg} \mathrm{ha}^{-1}$.

O acúmulo de $\mathrm{N}$ obtido foi, em média, 208,1 kg ha-1, abaixo daqueles encontrados por Alves et al. (2004), na mesma região, porém com faixas duplas (283 kg ha-1 de N). Neste trabalho, o P atingiu média de $8,16 \mathrm{~kg} \mathrm{ha}^{-1}$, e o acúmulo foi maior do que os $23 \mathrm{~kg} \mathrm{ha}^{-1}$ de P no experimento de faixas duplas citado. Segundo aquele trabalho, balanços positivos de N e P ocorrem quando o guandu em faixas duplas é utilizado em sistemas de produção de hortaliças. As quantidades de $\mathrm{N}$ e $\mathrm{P}$ acumuladas em guandu, em faixas simples, obtidas neste trabalho, seriam ainda suficientes para manter o balanço positivo de N e P (Alves et al., 2004). Em relação ao $\mathrm{P}$ e ao $\mathrm{K}$, não houve diferença entre genótipos.

Não houve diferença significativa entre as taxas de decomposição da fitomassa dos seis genótipos de guandu (Figura 1). Aproximadamente 75\% da fitomassa ainda

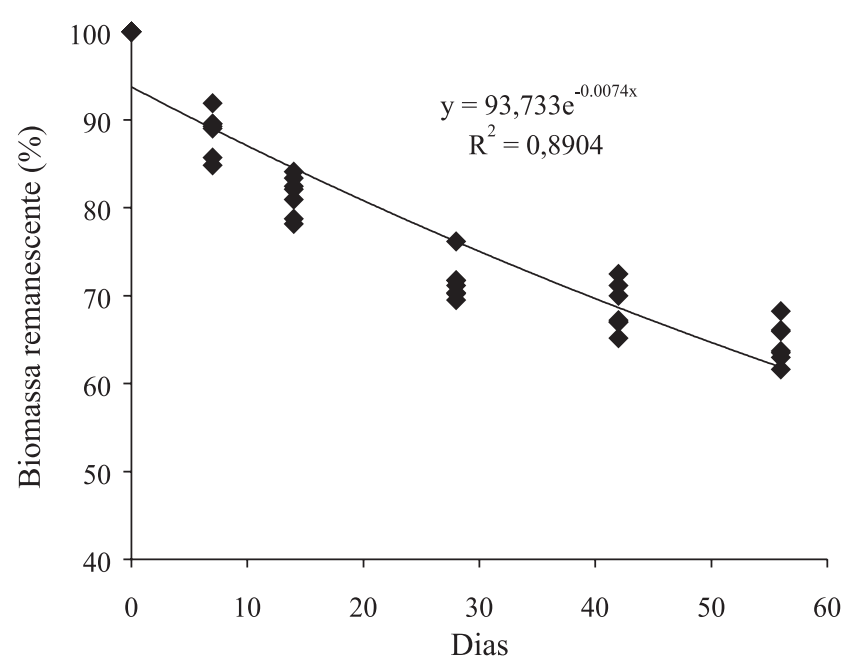

Figura 1. Taxa média de decomposição da biomassa de diferentes genótipos de guandu, depois de depositada na superfície do solo, na época do florescimento. 
restavam sobre o solo, aos 30 dias depois da deposição. Isto acarretou ao sistema uma boa cobertura do solo. Essa proteção ao solo, nos primeiros 30 dias depois do corte, coincide com o período mais crítico, caso uma lavoura comercial seja implantada entre as faixas, diminuindo, assim, a competição com plantas daninhas e favorecendo a conservação da umidade do solo. A constante de mineralização de nutrientes apresentou o padrão $\mathrm{K}>\mathrm{P}>\mathrm{N}$ (Figura 2). Ao final do primeiro mês, depois da colocação das bolsas de decomposição no campo, aproximadamente $60 \%$ do $\mathrm{N}$ contido, inicialmente, na fitomassa dos genótipos de guandu, ainda permaneciam na matéria seca, contra $40 \%$ que haviam sido liberados. Isso equivaleria à liberação de aproximadamente $80 \mathrm{~kg} \mathrm{ha}^{-1}$ de $\mathrm{N}$, se considerar a média de 208,1 $\mathrm{kg} \mathrm{ha}^{-1}$ na fitomassa produzida (Figura 1). Esses resultados confirmam os obtidos, em condições de campo, por Wagger (1989), Amado et al. (2000) e Aita et al. (2001).

Aita \& Giacomini (2003), ao avaliar a dinâmica da decomposição e liberação de $\mathrm{N}$ de resíduos culturais, observaram que enquanto em ervilhaca menos de $60 \%$ do N permaneceu nos resíduos culturais, nos primeiros 15 dias, no nabo e na aveia esse valor foi superior a $90 \%$.

Cerca de 50 e $45 \%$ de $\mathrm{P}$ e K, respectivamente, contidos na biomassa inicial dos genótipos de guandu, ainda permaneciam na matéria seca sobre a superfície do solo, aos 30 dias depois da colocação das bolsas, equivalentes a 4,0 $\mathrm{kg} \mathrm{ha}^{-1}$ de P e $19 \mathrm{~kg} \mathrm{ha}^{-1}$ de K liberados até os 30 dias, considerando-se os valores médios de $8,20 \mathrm{~kg} \mathrm{ha}^{-1}$ de Pe 34,10 $\mathrm{kg} \mathrm{ha}^{-1}$ de K (Tabela 2). Esses resultados estão de acordo com os dados obtidos por Da Ros (1993), que avaliou a liberação de P, durante a decomposição de diversas plantas de cobertura, e verificou que $64 \%$ do P da ervilhaca e $33 \%$ do P da aveia foram liberados nos primeiros 30 dias depois do manejo das plantas com uma gradagem.

Segundo Buchanan \& King (1993), a rápida liberação de P no período inicial da decomposição dos resíduos culturais está ligada à perda do P solúvel em água. A maior parte do $\mathrm{P}$ do tecido vegetal encontra-se no vacúolo da célula, na forma mineral, bastante solúvel em água (Marschner, 1995), e é liberado dos resíduos culturais quando o vacúolo é rompido (Aita \& Giacomini, 2003).

A liberação mais acelerada do P dos resíduos culturais, em relação ao $\mathrm{N}$, na fase inicial de decomposição, pode contribuir para aumentar a eficiência do P fornecido pelas plantas de cobertura, às culturas em suces- são, pelo fato de se aproximar melhor da curva de absorção de P pelas culturas, diminuindo tanto a sua imobilização pela população microbiana quanto a sua fixação no solo pelos óxidos de ferro (Frossard et al., 1995).

A rápida liberação de $\mathrm{K}$, ocorrida nos primeiros dias, confirma os resultados de Da Ros (1993), Schomberg \& Steiner (1999) e Moreira (2003), e pode ser atribuída ao fato de o K ser um elemento que não está associado a nenhum componente estrutural do tecido vegetal (Marschner, 1995). Schomberg \& Steiner (1999) observaram que, com precipitações elevadas, houve maior liberação de K nas leguminosas do que nas gramíneas. Segundo esses autores, mecanismos físicos (chuva) e químicos (qualidade do resíduo) estão envolvidos na liberação do nutriente, e em razão da elevada taxa de liberação de K dos resíduos culturais, parte dele poderá ser perdido no solo por lixiviação. Nesse sentido, o K talvez seja o elemento cuja redução do prazo de implantação das culturas em sucessão seja mais importante, para se minimizar perdas. A rápida liberação do K do tecido vegetal resultou num tempo de meia-vida menor que para o $\mathrm{N}$ e o $\mathrm{P}$.

Todos os genótipos avaliados apresentaram comportamento semelhante, em relação à dinâmica de liberação de N, P e K no processo de decomposição dos resíduos culturais. A liberação desses nutrientes, acumulados na biomassa, não chegou ao fim durante o período

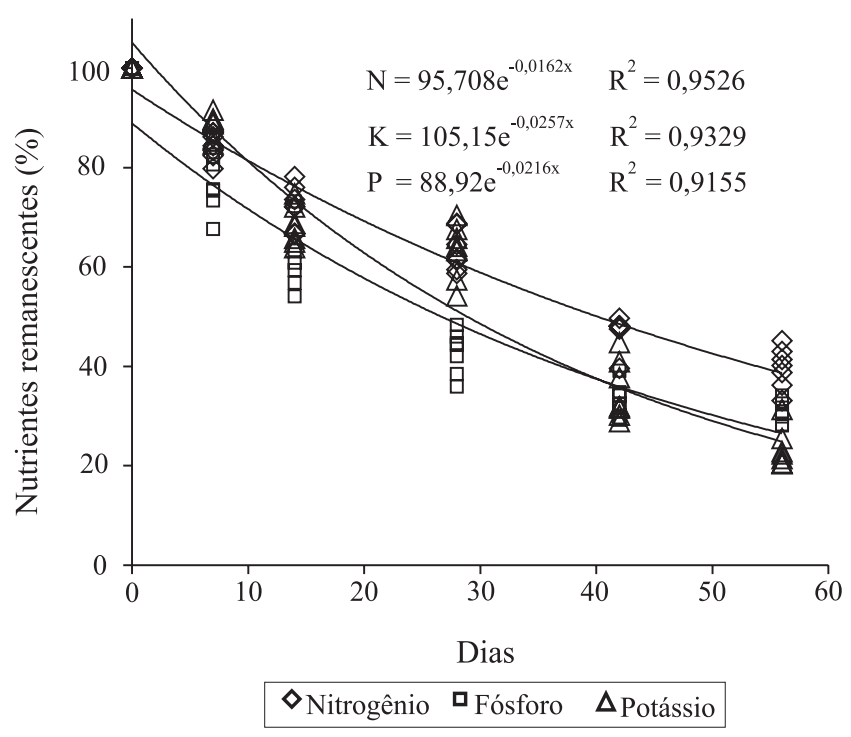

Figura 2. Evolução da mineralização de N, P e K, da biomassa dos diferentes genótipos de guandu, depois de depositada na superfície do solo, na época do florescimento. 
compreendido neste experimento. Na última avaliação, aos 56 dias, observou-se que, nesta época, aproximadamente $45 \%$ do $\mathrm{N}$ e $35 \%$ do $\mathrm{P}$ e do $\mathrm{K}$, contidos na biomassa remanescente, ainda não haviam sido liberados no solo (Figura 2). Todavia, considerando-se a elevada taxa de liberação do N, P e K das leguminosas, logo depois do seu manejo, é importante a busca de estratégias para maximizar o aproveitamento de nutrientes pelas culturas comerciais.

O conhecimento da dinâmica de liberação dos nutrientes é fundamental, para que as plantas que compõem as aléias possam ser eficientemente introduzidas no sistema de produção. É necessário, além disso, compatibilizar a máxima persistência dos resíduos culturais, na superfície do solo, com o fornecimento adequado de nutrientes, principalmente de N, para a cultura de interesse econômico, já que o material oriundo da poda preserva maior quantidade de resíduos culturais sobre o solo e contribui para a manutenção da umidade e para a proteção do solo contra o efeito erosivo da chuva.

Os resultados relativos à liberação de N, P e K indicaram que, em condições climáticas favoráveis à decomposição dos resíduos culturais das plantas de cobertura, é preciso otimizar a sincronia entre a liberação de nutrientes e a demanda pelas culturas comerciais. Mesmo no sistema de cultivo em aléias, em que os resíduos culturais permanecem na superfície do solo depois da poda, a liberação de nutrientes, na fase inicial de decomposição, pode superar a quantidade de nutrientes acumulados pelas culturas comerciais. Nas duas últimas décadas, foram realizados diversos trabalhos com plantas de cobertura de solo no outono/inverno, tanto no Brasil quanto em outros países, procurando-se caracterizar a decomposição e a liberação de $\mathrm{N}$ de seus resíduos culturais (Wagger, 1989; Ranells \& Wagger, 1992; Da Ros, 1993; Aita \& Giacomini, 2003). Esses estudos evidenciaram que as leguminosas, especialmente quando usadas como culturas solteiras, foram rapidamente decompostas depois do seu manejo, mesmo em sistemas de plantio direto, o que resultou num assincronismo entre a liberação de nutrientes dos seus resíduos culturais e a demanda de nutrientes pela cultura em sucessão. A elevada taxa de decomposição das leguminosas, resultante da baixa relação $\mathrm{C} / \mathrm{N}$ de seus resíduos, também contribui para diminuir a sua eficiência na manutenção da umidade e na proteção do solo contra a erosão (Derpsch et al., 1985).

\section{Conclusões}

1. Os seis genótipos de guandu não se diferenciam em relação à produtividade de biomassa e ao acúmulo de nutrientes na biomassa.

2. A taxa de liberação dos nutrientes de todos os genótipos apresenta padrões semelhantes, sendo maior para o K e menor para o $\mathrm{N}$.

\section{Agradecimentos}

À Embrapa Semi-Árido e ao Dr. Carlos Antônio Fernandes Santos, pela doação do germoplasma.

\section{Referências}

AITA, C.; BASSO, C.J.; CERETTA, C.A.; GONÇALVES, C.N.; DA ROS, C.O. Plantas de cobertura de solo como fontes de nitrogênio ao milho. Revista Brasileira de Ciência do Solo, v.25, p.157-165, 2001.

AITA, C.; GIACOMINI, S.J. Decomposição e liberação de nitrogênio de resíduos culturais de plantas de cobertura de solo solteiras e consorciadas. Revista Brasileira de Ciência do Solo, v.27, p.601612, 2003.

AKINOLA, J.O.; WHITEMAN, P.C. Agronomic studies on pigeon pea (Cajanus cajan (L.) Millsp.). I. Field responses to sowing time. Australian Journal of Agricultural Research, v.26, p.43-56, 1975.

ALVES, S.M.C.; ABBOUD, A.C. de S.; RIBEIRO, R. de L.D.; ALMEIDA, D.L. de. Balanço do nitrogênio e fósforo em solo com cultivo orgânico de hortaliças após a incorporação de biomassa de guandu. Pesquisa Agropecuária Brasileira, v.39, p.1111-1117, 2004.

AMADO, T.J.C.; MIELNICZUK, J.; FERNANDES, S.B.V. Leguminosas e adubação mineral como fontes de nitrogênio para o milho em sistemas de preparo do solo. Revista Brasileira de Ciência do Solo, v.24, p.179-189, 2000.

ANDERSON J.M.; INGRAM, J.S.I. (Ed.). Tropical soil biology and fertility: a handbook of methods. $2^{\text {nd }}$ ed. New York: Oxford, 1996. p.36-40.

BAHAR, F.A.; PRINE, G.M. Two cutting heights on ten pigeon peas (Cajanus cajan (L. Millsp.) harvested for foliage. Soil and Crop Science Society of Florida, v.41, p.144-148, 1982.

BUCHANAN, M.; KING, L. Carbon and phosphorus losses from decomposing crop residues in no-till and conventional till agroecosystems. Agronomy Journal, v.85, p.631-638, 1993.

DA ROS, C.O. Plantas de inverno para cobertura do solo e fornecimento de nitrogênio ao milho em plantio direto. 1993. 85p. Dissertação (Mestrado) - Universidade Federal de Santa Maria, Santa Maria.

DERPSCH, R.; SIDIRAS, N.; HEINZMANN, F.X. Manejo do solo com coberturas verdes de inverno. Pesquisa Agropecuária Brasileira, v.20, p.761-773, 1985. 
EMBRAPA. Serviço Nacional de Levantamento de Conservação de Solos (Rio de Janeiro, RJ). Manual de métodos e análise de solo. Rio de Janeiro, 1979. Não paginado.

FROSSARD, E.; BROSSARD, M.; HEDLEY, M.J.; MATHERELL, A. Reactions controlling the cycling of P in soil. In: TIESSEM, $\mathrm{H}$. (Org.). Phosphorus in the global environment: transfers, cycles and management. Chichester: J. Wiley, 1995. p.107-146.

KANG, B.T.; WILSON, G.F.; SIPKENS, L. Alley cropping maize and leucaena in Southern Nigeria. Plant and Soil, v.63, p.165-179, 1981.

MARSCHNER, H. Functions of mineral nutrients: macronutrients. In: MARSCHNER, H. Mineral nutrition of higher plants. $2^{\text {nd }}$ ed. San Diego: Academic, 1995. p.229-312.

MOREIRA, V.F. Produção de biomassa de guandu a partir de diferentes densidades de plantio e cultivo de brócolos em faixas intercalares sob manejo orgânico. 2003. 66p. Dissertação (Mestrado) - Universidade Federal Rural do Rio de Janeiro, Seropédica.

OTERO, J.R. de. Informações sobre algumas plantas forrageiras. 2.ed. Rio de Janeiro: Serviço de Informação Agrícola, 1961. 334p.

RANELLS, N.N.; WAGGER, M.G. Nitrogen release from crimson clover in relation to plant growth stage and composition. Agronomy Journal, v.84, p.424-430, 1992.
SCHOMBERG, H.H.; STEINER, J.L. Nutrient dynamics of crop residues decomposing on a fallow no-till soil surface. Soil Science Society of America Journal, v.63, p.607-613, 1999.

SILVA, M.B. Caracterização dos solos em uma topossequência no Campus da UFRRJ. 1993. 141p. Dissertação (Mestrado) Universidade Federal Rural do Rio de Janeiro, Seropédica.

SOUZA, J.L. de; RESENDE, P. Manual de horticultura orgânica. 1.ed. Viçosa: Aprenda Fácil, 2003. 554p.

TEDESCO, M.J.; GIANELLO, C.; BISSANI, C.A.; BOHNEN, H.; VOLKWEISS, S.J. Análise de solo, plantas e outros materiais. Porto Alegre: UFRGS, 1995. 174p.

THOMAS, R.J.; ASAKAWA, N.M. Decomposition of leaf litter from tropical forage grasses and legumes. Soil Biology and Biochemistry, v.25, p.1351-1361, 1993.

VIANA, O.J.; ALBUQUERQUE, J.J.L. Influência de diferentes tipos de cortes na produção de massa verde e sobrevivência do feijãoguandu, Cajanus flavus D. C. Boletim Cearense de Agronomia, v.10, p.23-36, 1969.

WAGGER, M.G. Time of desiccation effects on plant composition and subsequent nitrogen release from several winter annual cover crops. Agronomy Journal, v.81, p.236-241, 1989.

WUTKE, E.B. Característica fenológica e avaliação agronômica de genótipos de guandu (Cajanus cajan (L.) Millsp.). 1987. 164p. Dissertação (Mestrado) - Universidade de São Paulo, Piracicaba.

Recebido em 19 de janeiro de 2005 e aprovado em 9 de agosto de 2005 